\title{
パラメータ同定における複素数領域モデル規範 適応システムの有効性
}

\author{
正員竹下隆晴 (岐阜高專) \\ 正員松 井信 行 (名古屋工大)
}

\section{Effectivity of Using Model Reference Adaptive System in the Field of Complex Numbers for Parameter Identification}

Takaharu Takeshita, Member (Gifu National College of Technology), Nobuyuki Matsui, Member (Nagoya Institute of Technology)

The model reference adaptive system (MRAS) is generally described in the field of real numbers. Therefore, the MRAS cannot be directly applicable to the system described by complex numbers such as three-phase ac motors.

In order to apply the MRAS to the discrete-time linear time-invariant system whose coefficients and variables are complex numbers, the authors expand the theory of the MRAS into the field of complex numbers. The hyperstability theory in the field of complex numbers is used to give the asymptotic stability of the suggested algorithm.

The effectiveness of the suggested algorithm is proved by simulation results for the parameter identification. The results show that the convergence time of the parameters using the proposed method can be reduced to one tenth compared with that using the MRAS in the field of real numbers.

キーワード：モデル規範適応システム (MRAS), パラメー夕同定, 複素数領域, 超安定論, 交流電動機

\section{1. まえがき}

モデル規範適応システム(MRAS) は一般に実数 領域で理論展開され，複素数領域については述べられ ていないょうに思われる。従って，交流電動機などの 三相交流機器を複素座標上で記述した方程式 ${ }^{(1)} に$ 対し てMRASの理論を直接適用することはできない。

本論文では，係数および変数が複素数で記述される 離散時間多変数の線形時不変システムに対して, MRAS の理論が適用できるように実数領域の MRAS のパラメー夕同定理論を複素数領域に㹡張している。 同定アルゴリズムとしては基本的な比例十積分アルゴ リズムを扱っている。複素数領域に拡張された MRASの安定性の証明は, 実数領域の超安定論から 導出した複素数領域の超安定論を用いて行っている。 複素数領域と実数領域の MRASの関倸を明らかに
するために，複素数領域で記述されるシステムに等価 な実数領域のシステムの一表現法を示している。両者 の表現を比較すると，実数領域ではパラメータ行列に 物理的に等しい要素が二つずつ存在するので, パラメ 一夕行列の要素数は複素数領域の 2 倍になる。従っ て, 実数領域の MRAS を用いてパラメータ同定シス テムを構成すると物理的に等しいパラメータは別々に 同定されるので，同定に要する計算は複雑になり不合 理である。これに対して複素数領域のMRASを用い ると, 実数領域の半分のパラメー夕数を同定すること になることから，同定アルゴリズムの簡単化，計算量 の削隇が図られると共にパラメー夕収束速度の改善も 期待できる。

実数領域㧍よび複菜数領域それぞれのパラメータ同 定のシミュレーションを行い, 複素数領域でパラメー 夕を同定することの有効性を示している。 


\section{2. 複秦同定アルゴリズム}

離散時間多変数の線形時不変複帻システム

$$
\boldsymbol{y}(k+1)=\overline{\boldsymbol{A}} \boldsymbol{y}(k)+\overline{\boldsymbol{B}} \boldsymbol{u}(k)
$$

OMRASに基づいたパラメータ同定法について考え る。たたし，一は複素数の共役を意味し，y(k)， $u(k)$ \& $n \times 10$ 出力, 入力行列， $\bar{A}, \bar{B}$ \& $n \times n \sigma$ 人 ラメータ行列でそれでれ

$$
\begin{aligned}
& \boldsymbol{y}(k)=\left[\begin{array}{cc}
y_{r_{1}}(k)+j y_{i 1}(k) \\
\vdots & \vdots \\
y_{m}(k)+j y_{i n}(k)
\end{array}\right] \\
& \boldsymbol{u}(k)=\left[\begin{array}{cc}
u_{r 1}(k)+j u_{i 1}(k) \\
\vdots & \vdots \\
u_{r n}(k)+j u_{i n}(k)
\end{array}\right] \\
& \boldsymbol{A}=\left[\begin{array}{cccc}
a_{r 11}+j a_{i 11} \cdots & a_{r 1 n}+j a_{i 1 n} \\
\vdots & \vdots & \vdots & \vdots \\
a_{m 1}+j a_{i n 1} & \cdots & a_{m n}+j a_{i n n}
\end{array}\right]
\end{aligned}
$$

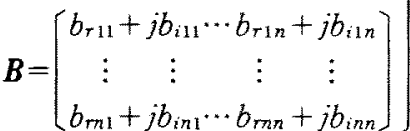

てある。

(1)式のプロセスのパラー夕行列 $\bar{A}, \bar{B}$ 㫫列 武MRASのアルゴりズムに上り同定する方法につい て説明する。(1)式のパラメータ行列と入出力行列在 专れ安れまとめて，

$$
\boldsymbol{y}(k+1)=\boldsymbol{P}^{*} \boldsymbol{\phi}(k)
$$

たたし，*：行列の其役軽置

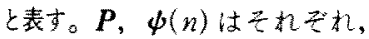

$$
P^{*}=\left[\begin{array}{ll}
\bar{A} & \bar{B}
\end{array}\right], \quad \boldsymbol{\phi}^{T}(k)=\left[\boldsymbol{y}^{T}(k) \boldsymbol{u}^{T}(k)\right]
$$

である。ここで，「は行列の転置を意味する。（1）式 のプロセスに対する推定もデル(2)を添字 $M$ を付けて，

$$
\begin{aligned}
\boldsymbol{y}_{M}(k+1) & =\overline{\boldsymbol{A}_{M}(k+1)} \boldsymbol{y}_{M}(k)+\overline{\boldsymbol{B}_{M}(k+1)} \boldsymbol{u}(k) \\
& =\boldsymbol{P} \text { 水 }(k+1) \boldsymbol{\phi}_{M}(k) \cdots \cdots \cdots \cdots(5)
\end{aligned}
$$

と定義する。ただし，推定パラメー夕行列 $\boldsymbol{P}_{M}^{*}(k+1)$, モデルの入出力行列 $\phi_{M}(k)$ 沬之れぞれ,

$$
\left.\begin{array}{l}
\boldsymbol{P}_{M}^{*}(k+1)=\left(\begin{array}{ll}
\boldsymbol{A}_{M}(k+1) & \overline{B_{M}(k+1)}
\end{array}\right] \\
\boldsymbol{\phi}_{M}^{T}(k)=\left[\begin{array}{ll}
\boldsymbol{y}_{M}^{T}(k) & \boldsymbol{u}^{T}(k)
\end{array}\right)
\end{array}\right\}
$$

でる。

プロセスと推定モデルの出力䛠蒫 $\boldsymbol{\varepsilon}(k)$ を,

$$
\varepsilon(k)=\boldsymbol{y}(k)-\boldsymbol{y}_{M}(k)
$$

と記し，次の(8) 〜 (11)式で与之られる比例+嫧分同 定アルゴリズムを用いることによって，実数領域の

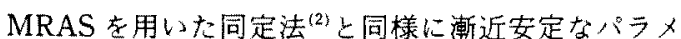
一夕同定システムが構成できる。

$$
\begin{aligned}
& \boldsymbol{P}_{M}(k)=\boldsymbol{P}_{M I}(k)+\boldsymbol{P}_{M P}(k) \cdots \cdots \cdots \cdots \cdots \cdot(8) \\
& \boldsymbol{P}_{M I}(k) \\
& \quad=\boldsymbol{P}_{M I}(k-1) \\
& \quad+\frac{\boldsymbol{F} \boldsymbol{\phi}_{M}(k-1) \boldsymbol{v}_{0}^{*}(k)}{1+\boldsymbol{\phi}_{M}^{*}(k-1)\left\{\boldsymbol{F}+\boldsymbol{F}^{\prime}(k-1)\right\} \boldsymbol{\phi}_{M}(k-1)}
\end{aligned}
$$

$$
\begin{aligned}
& \boldsymbol{P}_{M P}(k) \\
& \quad=\frac{\boldsymbol{F}^{\prime}(k-1) \boldsymbol{\phi}_{M}(k-1) \boldsymbol{v}_{0}^{*}(k)}{1+\boldsymbol{\phi}_{M}^{*}(k-1)\left\{\boldsymbol{F}+\boldsymbol{F}^{\prime}(k-1)\right\} \boldsymbol{\phi}_{M}(k-1)}
\end{aligned}
$$

$\boldsymbol{v}_{0}(k)=\boldsymbol{y}(k)-\boldsymbol{P}_{M I}^{*}(k-1) \boldsymbol{\phi}_{M}(k-1)$

$$
+C \varepsilon(k-1)
$$

ただし，Fは任意の正定エルミート行列 $(\boldsymbol{F}>0$, $\left.\boldsymbol{F}=\boldsymbol{F}^{*}\right)$ で, $\boldsymbol{F}^{\prime}(k)$ は任意のkに対して，

$$
\boldsymbol{F}^{\prime}(k)+1 / 2 \boldsymbol{F} \geqq 0
$$

在満足するエルミート行列である。また $n \times n$ 行列 $C$ はパルス伝達関数 $\boldsymbol{H}(z)$

$$
\boldsymbol{H}(z)=\left(\boldsymbol{I}_{n}+z^{-1} \boldsymbol{C}\right)\left(\boldsymbol{I}_{n}-z^{-1} \overline{\boldsymbol{A}}\right)^{-1}
$$

ただし， $I_{n}: n \times n の$ 算位行列

が，実数領域の強正害に相当した次の条件（I）， 満足するように定められる。

(I) $H(z)$ すへての要素が開平面 $|z| \geqq 1$ て解析 的七古こ二。

(II) $\boldsymbol{H}(z)+\boldsymbol{H}^{*}(z)$ 少単位平面上 $|z|=1$ て正定工 ルミート行列であること。

\section{3. 複素超安定論}

〈3.1〉複素数領域の超安定諭 (8) (11)式の 同定アルゴリズムを用いた同定システムは漸近安定と なること，すなわ次の(14)式が成立することを証明 するために、離散時間系の複素シスデムを前提とした 超安定論を示す。

$$
\lim _{k \rightarrow \infty} v(k)=0
$$

ただし，同定䛊差信号 $v(k)$ は $n \times 10$ 複素行列で，

$$
\begin{aligned}
v(k) & =\left[\begin{array}{cc}
v_{r 1}(k)+j v_{i 1}(k) \\
\vdots & \vdots \\
v_{m}(k)+j v_{i n}(k)
\end{array}\right] \\
& =\varepsilon(k)+C \varepsilon(k-1)
\end{aligned}
$$

てるる

図 1 性複素数を要素とする行列を同いた非線形時変 フィードック系でる。ここで, $\boldsymbol{w}(k), \boldsymbol{w}_{3}(k)$ は $n \times 1$ 複蓄行列で, 


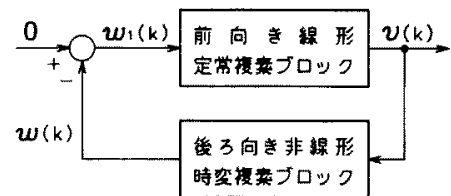

図 1 複素数領域の非線形時変 フィードバック系

Fig. 1. Nonlinear time-varying feedback system in the field of complex numbers.

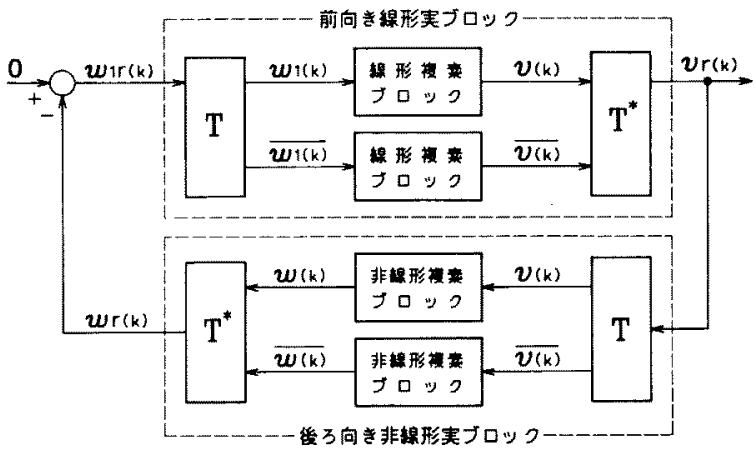

図 2 実数領域の非線形時変フィードバック系

Fig. 2. Nonlinear time-varying feedback system in the field of real numbers.

$$
\boldsymbol{w}(k)=\left[\begin{array}{cc}
w_{r 1}(k)+j w_{i 1}(k) \\
\vdots & \vdots \\
w_{r n}(k)+j w_{i n}(k)
\end{array}\right]=-w_{1}(k) \cdots(
$$

と記せる。このフィードバック系が漸近超安定となる 条件は，次の(1)，(2)をともに満足することである。

（1）前向き線形定常複素ブロックのパルス伝達関 数行列 $\boldsymbol{H}(z)$ が前章に示した条件（I），（II）を満足す ること。

（2）後ろ向き非線形時変複素ブロックが任意の $k_{1}$ に対して次式を満足すること。

$$
\sum_{k=0}^{k_{1}}\left\{\boldsymbol{v}^{*}(k) \boldsymbol{w}(k)+\boldsymbol{w}^{*}(k) v(k)\right\} \geqq-\gamma_{0}^{2}
$$

ただし， $\gamma_{0}^{2} は k_{1} に$ 依存しない有限な正の 定数

〈3・2〉実数領域の非線形フィードバック系

複素数領域の超安定論の証明を行うために, 図 $1 の$ 複菜数領域の非線形フィードバック系と図 2 の実数領 域の非線形フィードバック系が等価であることを示 す。図 2 の前向き線形ブロックは，図 1 の前向きブロ ックとその共役なブロックを並列にもち，更に $2 n \times$ $2 n$ のニニタリー変換行列 $\boldsymbol{T}$

$$
\boldsymbol{T}=\frac{1}{\sqrt{2}}\left[\begin{array}{rrrrrrr}
1 & j & 0 & 0 & \cdots & 0 & 0 \\
0 & 0 & 1 & j & \cdots & 0 & 0 \\
\vdots & \vdots & \vdots & \vdots & & \vdots & \vdots \\
0 & 0 & 0 & 0 & \cdots & 1 & j \\
1 & -j & 0 & 0 & \cdots & 0 & 0 \\
0 & 0 & 1 & -j & \cdots & 0 & 0 \\
\vdots & \vdots & \vdots & \vdots & & \vdots & \vdots \\
0 & 0 & 0 & 0 & \cdots & 1 & -j
\end{array}\right]
$$

を用いて構成され， $2 n \times 1$ の実行列 $\boldsymbol{v}_{r}(k)$ は，

$$
\begin{aligned}
& \boldsymbol{v}_{r}(k)=\boldsymbol{T}^{*}\left[\frac{\boldsymbol{v}(k)}{\boldsymbol{v}(k)}\right] \\
& =\sqrt{2}\left[v_{r 1}(k) v_{i 1}(k) \cdots v_{r n}(k) v_{i n}(k)\right]^{T}
\end{aligned}
$$

と得られる。図 2 の後ろ向き非線形ブロックも，図1 の後ろ向きブロックとその共役ブロックおよひ変換行 列 $\boldsymbol{T}$ により構成され， $2 n \times 1$ の実行列 $\boldsymbol{w}_{r}(k)$ は,

$$
\begin{aligned}
\boldsymbol{w}_{r}(k) & =-\boldsymbol{w}_{1 r}(k)=\boldsymbol{T}^{*}\left[\frac{\boldsymbol{w}(k)}{\boldsymbol{w}(k)}\right] \\
& =\sqrt{2}\left(w_{r 1}(k) w_{i 1}(k) \cdots w_{r n}(k) w_{i n}(k)\right]^{T}
\end{aligned}
$$

と得られる。（15），(16），(19），(20)式から明らかな ように $\boldsymbol{v}_{\boldsymbol{r}}(k), \boldsymbol{w}_{\boldsymbol{r}}(k)$ の各要素は $\boldsymbol{v}(k), \boldsymbol{w}(k)$ の各要 素の実部括び虚部を $\sqrt{2}$ 倍した值であり，図１と 図 2 のシステムは等価である。

また, 図 2 の線形ブロックの伝達関数行列 $\boldsymbol{H}_{r}(z)$ は,

$$
H_{r}(z)=T^{*}\left[\begin{array}{cc}
H(z) & 0 \\
0 & \bar{H}(\bar{z})
\end{array}\right] T
$$

と得られる。

〈3・3〉複素超安定論の証明 〈3・1〉節に示した複 素数領域の超安定論の証明を実数領域の超安定論に基 づいて行う。前節の説明から明らかなように図 1 と図 2 の系は等価であることから，図 2 の系が実数領域で 漸近超安定となる条件が，先に示した図2の複素数領 域の濑近超安定の条件 (1)，(2) と等価であることを 示す。

図 2 の非線形フィードバック系が漸近超安定である ためには，次の実数領域の超安定論の条件 (3)，(4) を満足しなければならない(2) (4)。

（3）前向き線形実ブロックのパルス伝達関数行列 $\boldsymbol{H}_{r}(z)$ が強正実であること。すなわち，次の条件 (III)，(IV)，(V)を同時に満足すること。

(III) $\boldsymbol{H}_{r}(z)$ のすべての要素が開平面 $|z| \geqq 1$ て解析 的であること。 
(IV) $|z| \geqq 1$ のすべての $z$ に対して $\overline{\boldsymbol{H}_{r}(z)}=\boldsymbol{H}_{r}(\bar{z})$ が成立すること。

(V) $\boldsymbol{H}_{r}(z)+\boldsymbol{H}_{r}^{T}(\bar{z})$ が単位平面上 $|z|=1$ で正定工 ルミート行列であること。

(4) 後ろ向き非線形時変実ブロックがすべての 衫対して次式を満足すること。

$$
\sum_{k=0}^{k_{1}} \boldsymbol{v}_{r}^{T}(k) \boldsymbol{w}_{r}(k) \geqq-\gamma_{1}^{2}
$$

ただし， $\gamma_{1}^{2} ： k_{1}$ に依存しない有限な正の定数

条件(3)，(4)が成り立つとき条件(1)，（2）が成 站ることを示す。

まず条件 (1)の（I）が成立することを示す。複素数 $h_{i j}(i, j=1,2, \cdots, m)$ を要素とする $m \times m$ 行列 $\boldsymbol{H}$ の， 山ム $\|\boldsymbol{H}\|$ を

$$
\|\boldsymbol{H}\|=\left\{\sum_{i=1}^{m} \sum_{j=1}^{m}\left|h_{i j}\right|^{2}\right\}^{1 / 2}
$$

と定義すれば，(21)式の関係加ら伝達関数行列 $H_{r}(z), H(z), \overline{H(\bar{z})}$ のそれぞれのノルムに対して,

$$
\left\|\boldsymbol{H}_{\mathrm{r}}(z)\right\|=\|\boldsymbol{H}(z)\|+\|\overline{H(\bar{z})}\|
$$

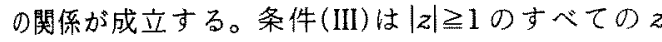
に対して $\left\|\boldsymbol{H}_{r}(z)\right\|$ が有限な值になることを意味してい る。このとき(24)式から明らかなように $0 \leqq\|\boldsymbol{H}(z)\| \leqq$ $\left\|H_{r}(z)\right\|$ であるから条件(1)の（I）は満足される。

次に，条件(1)の(II)が成立することを示す。 $H(z)+H^{*}(z)$ は明らかにエルミート行列であるから $|z|=1$ で正定であることを示せばよい。エルミート行 列が正定であるための必要十分条件はその行列のすべ ての固有值が正であることである(5)。また，(21)式 より,

$$
\begin{aligned}
& \boldsymbol{H}_{r}(z)+\boldsymbol{H}_{r}^{T}(\bar{z}) \\
& \quad=\boldsymbol{T}^{*}\left[\begin{array}{cc}
\boldsymbol{H}(z)+\boldsymbol{H}^{*}(z) & \mathbf{0} \\
\mathbf{0} & \overline{H(\bar{z})}+\boldsymbol{H}^{\tau}(\bar{z})
\end{array}\right] \boldsymbol{T}
\end{aligned}
$$

が成立する。従って，条件( 3 )の(V)上り $|z|=1$ に おいて $\boldsymbol{H}_{r}(z)+\boldsymbol{H}_{r}^{T}(\bar{z})$ のすべての固有值は正であり， その固有值は $\boldsymbol{T}$ がユニタリ行列であることから $\boldsymbol{H}(z)+\boldsymbol{H}^{*}(z)$ と $\overline{\boldsymbol{H}(\bar{z})}+\boldsymbol{H}^{T}(\bar{z})$ の固有值を合せたも のに等しいので(5)，条件(1)の(II)は満足される。

条件 (2)が成立することを示す。(19)，(20)式より 次の式変形ができる。

$$
\begin{aligned}
\boldsymbol{v}_{\tau}^{T}(k) \boldsymbol{w}_{r}(k) & =\left\{\boldsymbol{T}^{*}\left[\frac{\boldsymbol{v}(k)}{\boldsymbol{v}(k)}\right]\right\}^{*} \boldsymbol{T}^{*}\left[\frac{\boldsymbol{w}(k)}{\boldsymbol{w}(k)}\right] \\
& =\boldsymbol{v}^{*}(k) \boldsymbol{w}(k)+\left\{\boldsymbol{v}^{T}(k) \overline{\boldsymbol{w}(k)}\right\}^{r} \\
& =\boldsymbol{v}^{*}(k) \boldsymbol{w}(k)+\boldsymbol{w}^{*}(k) \boldsymbol{v}(k)
\end{aligned}
$$

従って，条件 (4)の(22)式より，

$$
\begin{aligned}
& \sum_{k=0}^{k_{1}} \boldsymbol{v}_{r}^{T}(k) \boldsymbol{w}_{r}(k) \\
& \quad=\sum_{k=0}^{k_{1}}\left\{\boldsymbol{v}^{*}(k) \boldsymbol{w}(k)+\boldsymbol{w}^{*}(k) \boldsymbol{v}(k)\right\} \geqq-\gamma_{1}^{2}
\end{aligned}
$$

が得られるので， $\gamma_{0}^{2}$ を $\gamma_{1}^{2} に$ 等しく選ぶことによって 条件 (2)が満足される。

逆に, 条件(1)，（2）が成り立つとき条件(3), (4)が成立することを示す。

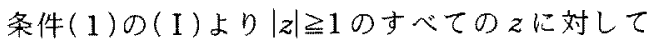
$\|\boldsymbol{H}(z)\|$ が有限な值になる。また $\overline{\boldsymbol{H}}(\bar{z})$ の極は $\boldsymbol{H}(z)$

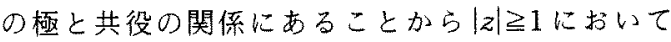
$\|\overline{H(Z \bar{z})}\|$ も有限な値になる。従って，(24)式より $\left\|\boldsymbol{H}_{r}(z)\right\|$ は $|z| \geqq 1$ において有限な值となることから条 件 ( 3 )の (III) が満足される。

\section{$\boldsymbol{T}^{T} \overline{\boldsymbol{T}}=\boldsymbol{I}_{2 n}$}

ただし， $\boldsymbol{I}_{2 n}: 2 n \times 2 n$ の単位行列

であることを考慮して，(21)式を変形すると，

$$
\begin{aligned}
\boldsymbol{H}_{r}(\bar{z})= & \boldsymbol{T}^{r} \overline{\boldsymbol{T}} \boldsymbol{T}^{*}\left[\begin{array}{cc}
\boldsymbol{H}(\bar{z}) & \mathbf{0} \\
\mathbf{0} & \overline{\boldsymbol{H}(z)}
\end{array}\right] \boldsymbol{T} \boldsymbol{T}^{r} \overline{\boldsymbol{T}} \\
= & \boldsymbol{T}^{r}\left[\begin{array}{cc}
\mathbf{0} & \boldsymbol{I}_{n} \\
\boldsymbol{I}_{n} & \mathbf{0}
\end{array}\right]\left[\begin{array}{cc}
\boldsymbol{H}(\bar{z}) & \mathbf{0} \\
\mathbf{0} & \overline{\boldsymbol{H}(z)}
\end{array}\right]\left[\begin{array}{cc}
\mathbf{0} & \boldsymbol{I}_{n} \\
\boldsymbol{I}_{n} & 0
\end{array}\right] \overline{\boldsymbol{T}} \\
= & \overline{\boldsymbol{T}^{*}\left[\begin{array}{cc}
\boldsymbol{H}(z) & \mathbf{0} \\
\mathbf{0} & \overline{\boldsymbol{H}(\bar{z})}
\end{array}\right] \boldsymbol{T}}=\overline{\boldsymbol{H}_{r}(z)}
\end{aligned}
$$

の関係が得られ，条件 (3)の(IV)が満足される。

先に述へたように，エルミート行列が正定であるた めの必要十分条件は, その行列のすべての固有值が正 であることである。条件(1)の(II)より $|z|=1$ で $\boldsymbol{H}(z)+\boldsymbol{H}^{*}(z)$ は正定であるから $z$ に対して共役な $\boldsymbol{H}(\bar{z})+\boldsymbol{H}^{*}(\bar{z})$ の固有值はすべて正である。更に, 全 体の共役をとった $\overline{\boldsymbol{H}(\bar{z})}+\boldsymbol{H}^{T}(\bar{z})$ の固有值もすべて 正となり，(25)式から明らかなように条件 (3)の(V) が満足される。

条件 (4)については，条件 (2)抢よび $(26)$ 式より任 意の $k_{1}$ に対して,

$$
\begin{gathered}
\sum_{k=0}^{k_{1}}\left\{\boldsymbol{v}^{*}(k) \boldsymbol{w}(k)+\boldsymbol{w}^{*}(k) \boldsymbol{v}(k)\right\} \\
=\sum_{k=0}^{k_{1}} \boldsymbol{v}_{r}^{T}(k) \boldsymbol{w}_{r}(k) \geqq-\gamma_{0}^{2} \cdots \cdots
\end{gathered}
$$

が成立するので， $\gamma_{1}^{2} を \gamma_{0}^{2} に$ 等しく選ぶことによって 満足される。

\section{4. 漸近安定の証明}

〈4・1〉等価フィードバック系の導出 第 2 章に示 した同定アルゴリズムを用いると同定システムが漸近 
安定となることを前章の複素超安定論を用いて証明す る。まず同定システムの等洒フィードバック系を導出 する。(1)，(5)，(7)式より次式が得られる。

$$
\boldsymbol{\varepsilon}(k+1)=\overline{\boldsymbol{A}} \boldsymbol{\varepsilon}(k)+\left\{\boldsymbol{P}-\boldsymbol{P}_{M}(k+1)\right\}^{*} \boldsymbol{\psi}_{M}(k)
$$

推定モデルの出力および出力誤差のそれぞれの暫定 值を,

$$
\begin{aligned}
& \boldsymbol{y}_{M 0}(k+1)=\boldsymbol{P}_{M I}^{*}(k) \boldsymbol{\psi}_{M}(k) \\
& \boldsymbol{\varepsilon}_{0}(k)=\boldsymbol{y}(k)-\boldsymbol{y}_{M 0}(k) \cdots \ldots
\end{aligned}
$$

と定義する。（1），(11)，(31)，(32)式より，

$$
\boldsymbol{\varepsilon}_{0}(k+1)=\overline{\boldsymbol{A}} \boldsymbol{\varepsilon}_{0}(k)+\left\{\boldsymbol{P}-\boldsymbol{P}_{M I}(k+1)\right\}^{*} \boldsymbol{\psi}_{M}(k)
$$

$$
\boldsymbol{v}_{0}(k)=\varepsilon_{0}(k)+\boldsymbol{C} \boldsymbol{\varepsilon}(k-1)
$$

が得られる。(15)式から(34)式を辺々差し引き, (30)，(33)式の関係を代入すると，

$$
\boldsymbol{v}(k)-\boldsymbol{v}_{0}(k)=\left\{\boldsymbol{P}_{M I}(k-1)-\boldsymbol{P}_{M}(k)\right\}^{*} \boldsymbol{\psi}_{M}(k-1)
$$

となり, 更に $(8) \sim(10)$ 式を用いて変形すると，

$$
v(k)
$$

$$
=\frac{\boldsymbol{v}_{0}(k)}{1+\boldsymbol{\psi}_{M}^{*}(k-1)\left\{\boldsymbol{F}+\boldsymbol{F}^{\prime}(k-1)\right\} \boldsymbol{\psi}_{M}(k-1)}
$$

が得られる。（9），(10)式は(36)式を用いてそれぞれ

$$
\boldsymbol{P}_{M I}(k)=\boldsymbol{P}_{M I}(k-1)+\boldsymbol{F} \boldsymbol{\psi}_{M}(k-1) \boldsymbol{v}^{*}(k)
$$

$$
\boldsymbol{P}_{M P}(k)=\boldsymbol{F}^{\prime}(k-1) \boldsymbol{\phi}_{M}(k-1) \boldsymbol{v}^{*}(k)
$$

と変形できる。

ここで, $\boldsymbol{r}(k), \boldsymbol{w}(k), \boldsymbol{w}_{1}(k)$ をそれぞれ，

$$
\begin{aligned}
& \boldsymbol{r}(k)=\boldsymbol{P}_{M}(k)-\boldsymbol{P} \ldots \ldots \ldots \ldots \ldots \ldots \ldots \ldots \ldots \\
& \boldsymbol{w}(k)=-\boldsymbol{w}_{1}(k)=\left\{\boldsymbol{P}_{M}(k)-\boldsymbol{P}\right\}^{*} \boldsymbol{\phi}_{M}(k-1)
\end{aligned}
$$

と定義すると，(8)，(15)，(30)，(37) 〜 (40) 式を用 いて等価フィードバック系が,

$$
\begin{aligned}
& \boldsymbol{\varepsilon}(k)=\overline{\boldsymbol{A}} \boldsymbol{\varepsilon}(k-1)+\boldsymbol{w}_{1}(k) \\
& \boldsymbol{v}(k)=\boldsymbol{\varepsilon}(k)+\boldsymbol{C} \boldsymbol{\varepsilon}(k-1) \\
& \boldsymbol{r}(k)=\boldsymbol{r}(k-1)+\boldsymbol{F} \boldsymbol{\phi}_{M}(k-1) \boldsymbol{v}^{*}(k) \\
& \boldsymbol{w}^{*}(k)=-\boldsymbol{w}_{1}^{*}(k) \\
& =\boldsymbol{\psi}_{M}^{*}(k-1) \boldsymbol{r}(k-1)+\boldsymbol{\psi}_{M}^{*}(k-1) \\
& \times\left\{\boldsymbol{F}+\boldsymbol{F}^{\prime}(k-1)\right\} \boldsymbol{\phi}_{M}(k-1) \boldsymbol{v}^{*}(k)
\end{aligned}
$$

と得られる。図 3 に示すように(41)，(42)式が前向き 線形複素ブロック，(43)，(44)式が後ろ向き非線形複 素ブロックをそれぞれ表す。

〈4・2〉安定性の証明＼cjkstart等価〉ィードバック系が 漸近超安定であるための条件 (1)，（2）を満足するこ

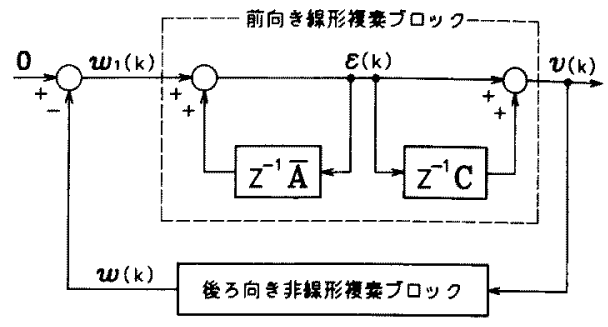

図 3 等価フィードバック系

Fig. 3. Equivalent feedback system.

とを示す。行列 $C$ は，(13)式の前向き線形ブロック のパルス伝達関数行列 $\boldsymbol{H}(z)$ が条件 (1)を満たすよう に決められるので, 後ろ向き非線形ブロックが条件 (2)を満足することを示せば十分である。(44)式の $\boldsymbol{w}(k)$ を次式のように $\boldsymbol{w}_{a}(k)$ と $\boldsymbol{w}_{b}(k)$ に分けて,

$$
\begin{aligned}
\boldsymbol{w}^{*}(k)= & \boldsymbol{w}_{a}^{*}(k)+\boldsymbol{w}_{b}^{*}(k) \quad \cdots \cdots \cdots \cdots \cdots(45) \\
\boldsymbol{w}_{a}^{*}(k)= & \boldsymbol{\psi}_{M}^{*}(k-1)\left\{1 / 2 \boldsymbol{F}+\boldsymbol{F}^{\prime}(k-1)\right\} \\
& \times \boldsymbol{\psi}_{M}(k-1) \boldsymbol{v}^{*}(k) \quad \cdots \cdots \cdots \cdots \cdots(46) \\
\boldsymbol{w}_{b}^{*}(k)= & \boldsymbol{\psi}_{M}^{*}(k-1) \boldsymbol{r}(k-1)+1 / 2 \boldsymbol{\psi}_{M}^{*}(k-1) \boldsymbol{F} \\
& \times \boldsymbol{\psi}_{M}(k-1) \boldsymbol{v}^{*}(k) \cdots \cdots \cdots \cdots \cdots(47)
\end{aligned}
$$

と表したとき，任意の $k_{1}$ に対して，

$$
\begin{aligned}
& \sum_{k=0}^{k_{1}}\left\{\boldsymbol{v}^{*}(k) \boldsymbol{w}_{a}(k)+\boldsymbol{w}_{a}^{*}(k) \boldsymbol{v}(k)\right\} \geqq-\gamma_{a}^{2} \cdots \\
& \sum_{k=0}^{k_{1}}\left\{\boldsymbol{v}^{*}(k) \boldsymbol{w}_{b}(k)+\boldsymbol{w}_{b}^{*}(k) \boldsymbol{v}(k)\right\} \geqq-\gamma_{b}^{2} \cdots
\end{aligned}
$$

を満足する $k_{1}$ に依存しない正の定数 $\gamma_{a}^{2}, \gamma_{b}^{2}$ が存在す れば条件 (2)は成立する。(12)式を考慮して(46)式を 用いて (48)式の左辺を計算すると，

$$
\begin{array}{r}
\sum_{k=0}^{k_{1}} 2 \boldsymbol{v}^{*}(k) \boldsymbol{v}(k) \boldsymbol{\psi}_{M}^{*}(k-1)\{1 / 2 \boldsymbol{F} \\
\left.\left.+\boldsymbol{F}^{\prime}(k-1)\right\} \boldsymbol{\psi}_{M}(k-1)\right\} \geqq 0
\end{array}
$$

となるので，(48)式は満足される。次の定理を用いて (49)式が成立することを説明する。

【定 理〕離散時間の非線形複素システム

$$
\begin{aligned}
& \boldsymbol{x}(k+1)=\boldsymbol{A}(k) \boldsymbol{x}(k)+\boldsymbol{B}(k) \boldsymbol{u}(k+1) \cdots(51) \\
& \boldsymbol{v}(k+1)=\boldsymbol{C}(k) \boldsymbol{x}(k)+\boldsymbol{D}(k) \boldsymbol{u}(k+1) \cdots(52)
\end{aligned}
$$

について以下の関係が成立する。ただし，すべての行 列はその要素が複菜数であり, $x(k)$ は $m_{1} \times m_{2}$, $\boldsymbol{u}(k)$ および $\boldsymbol{v}(k)$ は $m_{3} \times m_{2}$ の行列であり, $\boldsymbol{A}(k)$, $\boldsymbol{B}(k), \boldsymbol{C}(k), \boldsymbol{D}(k)$ はそれぞれ適切な次元の時変行 列である。(51)，(52)式のシステムにおいて，

$$
\begin{array}{r}
\boldsymbol{A}^{*}(k) \boldsymbol{P}(k+1) \boldsymbol{A}(k)-\boldsymbol{P}(k)=-\boldsymbol{Q}(k) \\
\ldots \ldots \ldots \ldots \ldots \ldots \ldots \ldots \ldots \ldots \ldots \ldots \ldots \ldots \ldots \\
\boldsymbol{B}^{*}(k) \boldsymbol{P}(k+1) \boldsymbol{A}(k)+\boldsymbol{S}^{*}(k)
\end{array}
$$




$$
\boldsymbol{D}(k)+\boldsymbol{D}^{*}(k)-\boldsymbol{B}^{*}(k) \boldsymbol{P}(k+1) \boldsymbol{B}(k)=\boldsymbol{R}(k)
$$

$$
\left[\begin{array}{ll}
\boldsymbol{Q}(k) & \boldsymbol{S}(k) \\
\boldsymbol{S}^{*}(k) & \boldsymbol{R}(k)
\end{array}\right] \geqq 0
$$

老满足する時変正定エルミ一ト行列 $\boldsymbol{P}(k)$, 時変半正 定エルミート行列 $\boldsymbol{Q}(k)$ おょび時変行列 $\boldsymbol{R}(k), \boldsymbol{S}(k)$ か存在するならば，任意の $k_{1}$ に対して不等式

$$
\begin{gathered}
\sum_{k=0}^{k_{1}} \operatorname{tr}\left\{\boldsymbol{v}^{*}(k+1) \boldsymbol{u}(k+1)+\boldsymbol{u}^{*}(k+1) \boldsymbol{v}(k+1)\right\} \\
\geqq-\operatorname{tr}\left\{\boldsymbol{x}^{*}(0) \boldsymbol{P}(0) \boldsymbol{x}(0)\right\} \quad \cdots \cdots \cdots \cdots \cdots(57)
\end{gathered}
$$

が満足される。ただし， tr は行列のトレースを意味 する。 (定理終)

上記の定理に用いている記号は, 本論文中の同じ記 号とは区別して用いられていることに注意しよう。ま た，定理の証明は付録に記した。

定理の (51)，(52) 式と (43)，(47)式を比較し，

$$
\left.\begin{array}{ll}
\boldsymbol{x}(k+1)=\boldsymbol{r}(k), & \boldsymbol{u}(k+1)=\boldsymbol{v}^{*}(k) \\
\boldsymbol{v}(k+1)=\boldsymbol{w}_{b}^{*}(k), & \boldsymbol{A}(k)=\boldsymbol{I}_{2 n} \\
\boldsymbol{B}(k)=\boldsymbol{F} \boldsymbol{\phi}_{M}(k-1), & \boldsymbol{C}(k)=\boldsymbol{\psi}_{M}^{*}(k-1) \\
\boldsymbol{D}(k)=1 / 2 \boldsymbol{\psi}_{M}^{*}(k-1) \boldsymbol{F} \boldsymbol{\psi}_{M}(k-1)
\end{array}\right\}
$$

と対応させる。ただし，上式の左辺は(51)，(52)式の 行列，右辺は(43)，(47)式の行列である。更に,

$$
\left.\begin{array}{l}
\boldsymbol{P}(k)=\boldsymbol{F}^{-1}(>0), \quad \boldsymbol{Q}(k)=\mathbf{0} \\
\boldsymbol{R}(k)=\mathbf{0}, \quad \boldsymbol{S}(k)=\mathbf{0}
\end{array}\right\}
$$

と選ふことで，定理の(53)〜(56)式がすべて满足され ろので，(57)式に対応して，

$$
\begin{gathered}
\sum_{k=0}^{k_{1}} \operatorname{tr}\left\{\boldsymbol{w}_{b}(k) \boldsymbol{v}^{*}(k)+\boldsymbol{v}(k) \boldsymbol{w}_{b}^{*}(k)\right\} \\
\quad=\sum_{k=0}^{k_{1}}\left\{\boldsymbol{v}^{*}(k) \boldsymbol{w}_{b}(k)+\boldsymbol{w}_{b}^{*}(k) \boldsymbol{v}(k)\right\} \\
\geqq-\operatorname{tr}\left\{\boldsymbol{r}^{*}(0) \boldsymbol{F}^{-1} \boldsymbol{r}(0)\right\} \ldots \ldots \ldots \ldots \ldots
\end{gathered}
$$

が任意のkにについて成立し，(49)式が満足される。 以上により等価フィードバック系は漸近安定となり， (14)式が成立することが示された。

\section{5. 実方程式への一変換法}

(1)式の複素システムに対灾した害システムについ て考える。(1)式とその共役な方程式

$$
\overline{\boldsymbol{y}(k+1)}=\boldsymbol{A} \overline{\boldsymbol{y}(k)}+\boldsymbol{B} \overline{\boldsymbol{u}(k)}
$$
を含せて，

$$
\left[\begin{array}{l}
\boldsymbol{y}(k+1) \\
\boldsymbol{y}(k+1)
\end{array}\right]=\left[\begin{array}{ll}
\overline{\boldsymbol{A}} & 0 \\
\mathbf{0} & \boldsymbol{A}
\end{array}\right]\left[\begin{array}{l}
\boldsymbol{y}(k) \\
\boldsymbol{y}(k)
\end{array}\right]+\left[\begin{array}{ll}
\overline{\boldsymbol{B}} & \boldsymbol{0} \\
\mathbf{0} & \boldsymbol{B}
\end{array}\right]\left[\begin{array}{l}
\boldsymbol{u}(k) \\
\overline{\boldsymbol{u}(k)}
\end{array}\right]
$$

が得られる。(62)式を(18)式のユ二タリ一行列 $\boldsymbol{T}$ を 用いて変換すると次の実方程式が得られる。

$$
\boldsymbol{y}_{r}(k+1)=\boldsymbol{A}_{r} \boldsymbol{y}_{r}(k)+\boldsymbol{B}_{r} \boldsymbol{u}_{r}(k)
$$

ただし， $\boldsymbol{y}_{r}(k), \boldsymbol{u}_{r}(k)$ はそれぞれ $2 n \times 1$ の出力, 入 力行列, $\boldsymbol{A}_{r}, \boldsymbol{B}_{r}$ は $2 n \times 2 n$ のパラメー夕行列,

$$
\begin{aligned}
& \boldsymbol{y}_{r}(k)=\boldsymbol{T}^{*}\left[\frac{\boldsymbol{y}(k)}{\boldsymbol{y}(k)}\right] \\
& =\sqrt{2}\left[y_{r 1}(k) y_{i 1}(k) \cdots y_{m}(k) y_{i n}(k)\right]^{T} \\
& \boldsymbol{u}_{r}(k)=\boldsymbol{T}^{*}\left[\frac{\boldsymbol{u}(k)}{\boldsymbol{u}(k)}\right] \\
& =\sqrt{2}\left[u_{r_{1}}(k) u_{i 1}(k) \cdots u_{r n}(k) u_{i n}(k)\right]^{T}
\end{aligned}
$$

$A_{r}=T^{*}\left[\begin{array}{ll}\bar{A} & 0 \\ 0 & A\end{array}\right] T$

$$
=\left[\begin{array}{cccccc}
a_{r 11} & a_{i 11} & a_{r 12} & \cdots & a_{r 1 n} & a_{i 1 n} \\
-a_{i 11} & a_{r 11} & -a_{i 12} & \cdots & -a_{r 1 n} & a_{i 1 n} \\
\vdots & \vdots & \vdots & & \vdots & \vdots \\
a_{m 1} & a_{i n 1} & a_{m 2} & \cdots & a_{r n n} & a_{i n n} \\
-a_{i n 1} & a_{m 1} & -a_{i n 2} & \cdots & -a_{i n n} & a_{m n}
\end{array}\right]
$$

$$
\begin{aligned}
\boldsymbol{B}_{r} & =\boldsymbol{T}^{*}\left[\begin{array}{cc}
\overline{\boldsymbol{B}} & \mathbf{0} \\
\boldsymbol{0} & \boldsymbol{B}
\end{array}\right] \boldsymbol{T} \\
& =\left[\begin{array}{cccccc}
b_{r 11} & b_{i 11} & b_{r 12} & \cdots & b_{r 1 n} & b_{i 1 n} \\
-b_{i 11} & b_{r 11} & -b_{i 12} & \cdots & -b_{r 1 n} & b_{i n n} \\
\vdots & \vdots & \vdots & & \vdots & \vdots \\
b_{m 11} & b_{i n 1} & b_{m 2} & \cdots & b_{m n} & b_{i n n} \\
-b_{i n 1} & b_{r n 1} & -b_{i n 2} & \cdots & -b_{i n n} & b_{m n}
\end{array}\right]
\end{aligned}
$$

である。（1），(2)式の複素方程式と(63)，(64)式の 実方程式を比較して明らかなように，複索方程式の各 要素の実, 虚数部は実方程式ではそれぞれ独立した一 つの要素に対応し，入出力行列の各要素は実方程式で は $\sqrt{2}$ 倍され，パラメータ行列については要素の大 きさは変化しない。従って，(63)式の実方程式は(1) 式の複素方程式と等価な方程式である。

(63)式の実方程式のパラメータ行列 $\boldsymbol{A}_{r}, \boldsymbol{B}_{r}$ の要 素において(64)式から明らかなように物理的に等しい ものが二つずつ存在する。徉って，赛数領域の MRASを用いて(63)式のパラメータを同定すると $8 n^{2}$ 個のパラメー夕を同定しなければならない。（1） 式の複素方程式に抢いてはパラメー夕行列 $\bar{A}, \bar{B} の$ 要素数结実部，虚部をそれぞれ一つのパラメータとし て报うと $4 n^{2}$ となり，実方程式の半分になっている。 このように同定するパラメータの数を隇少することに より，同定アルゴリズムの簡単化，同定処理時間の短 縮抢よびパラメータの収束速度の改善が期待できる。 


\section{6. シミュレーション結果}

複素数領域で同定することの有効性を示すために， 簡単な一例に対して複素数領域括よび実数領域それぞ れのパラメー夕同定のシミュレーションを行う。

$\langle 6 \cdot 1\rangle$ 複素数領域の同定特性 (1)式の方程式 とその入力がそれぞれ

$$
\begin{aligned}
& y_{1}(k+1)+j y_{2}(k+1) \\
& \quad=[0.8-j 0.51-j 0.3]\left[\begin{array}{l}
y_{1}(k)+j y_{2}(k) \\
u_{1}(k)+j u_{2}(k)
\end{array}\right]
\end{aligned}
$$

$$
\begin{aligned}
u_{1}(k) & +j u_{2}(k) \\
= & \cos (2 \pi k / 10)+0.8 \cos (2 \pi k / 8) \\
& +0.6 \cos (2 \pi k / 6)+0.4 \cos (2 \pi k / 4) \\
& +j\{\sin (2 \pi k / 10)+0.8 \sin (2 \pi k / 8) \\
& +0.6 \sin (2 \pi k / 6)+0.4 \sin (2 \pi k / 4)\}
\end{aligned}
$$

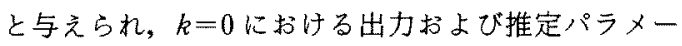
夕を零としてパラメー夕同定を行ったときの特性を求 める。（5），(31）式にそれぞれ対応した出力とその暫 定值は,

$$
\begin{aligned}
& y_{M 1}(k+1)+j y_{M 2}(k+1) \\
& =\left[a_{M 1}(k+1)-j a_{M 2}(k+1) b_{M 1}(k+1)\right. \\
& \left.-j b_{M 2}(k+1)\right)\left[\begin{array}{l}
y_{M 1}(k)+j y_{M 2}(k) \\
u_{1}(k)+j u_{2}(k)
\end{array}\right] \\
& y_{M 01}(k+1)+j y_{M 02}(k+1) \\
& =\left[a_{M 1}(k)-j a_{M 2}(k) \quad b_{M 1}(k)-j b_{M 2}(k)\right] \\
& \times\left[\begin{array}{l}
y_{M 1}(k)+j y_{M 2}(k) \\
u_{1}(k)+j u_{2}(k)
\end{array}\right]
\end{aligned}
$$

と記せる。また $(8) \sim(11)$ 式の同定アルゴリズムにお いて $\boldsymbol{F}=5 \boldsymbol{I}_{n}, \boldsymbol{F}^{\prime}(k)=\mathbf{0}, \boldsymbol{C}=\mathbf{0}$ と選ぶと, 次の同定 アルゴリズムが得られる。

$$
\begin{aligned}
& {\left[\begin{array}{l}
a_{M 1}(k)+j a_{M 2}(k) \\
b_{M 1}(k)+j b_{M 2}(k)
\end{array}\right]} \\
& =\left[\begin{array}{l}
a_{M 1}(k-1)+j a_{M 2}(k-1) \\
b_{M 1}(k-1)+j b_{M 2}(k-1)
\end{array}\right] \\
& \quad \times 5\left[\begin{array}{l}
y_{M 1}(k-1)+j y_{M 2}(k-1) \\
u_{1}(k-1)+j u_{2}(k-1)
\end{array}\right]\left\{\varepsilon_{01}(k)\right. \\
& \left.\quad-j \varepsilon_{02}(k)\right\} /\left[1+5\left\{y_{M 1}^{2}(k-1)+y_{M 2}^{2}(k-1)\right.\right. \\
& \left.\left.\quad+u_{1}^{2}(k-1)+u_{2}^{2}(k-1)\right\}\right] \ldots \ldots \ldots(69) \\
& \varepsilon_{01}(k)+j \varepsilon_{02}(k) \\
& =\left\{y_{1}(k)-y_{M 01}(k)\right\}+j\left\{y_{2}(k)-y_{M 02}(k)\right\}
\end{aligned}
$$

ただし

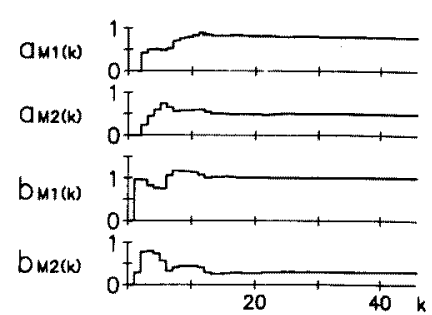

図 4 複菜同定アルゴリズムによる同定特性 Fig. 4. Identification characteristics based on the proposed complex identification algorithm.

である。図 4 に上記の同定アルゴリズムに従ってパラ メー夕同定を行ったときの特性を示す。25サンプル 目の推定パラメータの值はそれぞれ $a_{M 1}(25)=0.801$, $a_{M 2}(25)=0.504, b_{M 1}(25)=0.998, b_{M 2}(25)=0.295 \tau$ あり,プロセスのパラメータにほとんど一致している。

〈6-2〉 実数領域での同定特性 (65)式の複素方 程式を第 5 章で述べたように実方程式に変更し, 参考 文献 (2)などに述べられている赛数領域の MRASを 用いてパラメー夕同定を行った場合の特性を求める。 (65)式に対応したプロセスとその推定モデルの出力お よび暫定值はそれぞれ，

$$
\begin{aligned}
{\left[\begin{array}{l}
\sqrt{2} y_{1}(k+1) \\
\sqrt{2} y_{2}(k+1)
\end{array}\right]=} & {\left[\begin{array}{rr}
0.8 & 0.5 \\
-0.5 & 0.8
\end{array}\right]\left[\begin{array}{l}
\sqrt{2} y_{1}(k) \\
\sqrt{2} y_{2}(k)
\end{array}\right] } \\
& +\left[\begin{array}{cc}
1 & 0.3 \\
-0.3 & 1
\end{array}\right]\left[\begin{array}{l}
\sqrt{2} u_{1}(k) \\
\sqrt{2} u_{2}(k)
\end{array}\right]
\end{aligned}
$$

$$
\begin{aligned}
& {\left[\begin{array}{l}
\sqrt{2} y_{M 1}(k+1) \\
\sqrt{2} y_{M 2}(k+1)
\end{array}\right]}
\end{aligned}
$$

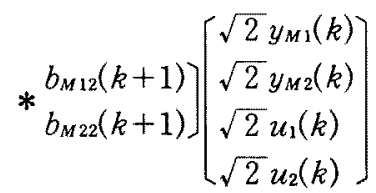$$
=\left[\begin{array}{lll}
a_{M 11}(k+1) & a_{M 12}(k+1) & b_{M 11}(k+1) \\
a_{M 21}(k+1) & a_{M 22}(k+1) & b_{M 21}(k+1)
\end{array} *\right.
$$

$$
\begin{aligned}
& {\left[\begin{array}{l}
\sqrt{2} y_{M 01}(k+1) \\
\sqrt{2}{ }_{M 02}(k+1)
\end{array}\right]} \\
& =\left[\begin{array}{llll}
a_{M 11}(k) & a_{M 12}(k) & b_{M 11}(k) & b_{M 12}(k) \\
a_{M 21}(k) & a_{M 22}(k) & b_{M 21}(k) & b_{M 22}(k)
\end{array}\right] \\
& \times\left[\begin{array}{l}
\sqrt{2} y_{M 1}(k) \\
\sqrt{2} y_{M 2}(k) \\
\sqrt{2} u_{1}(k) \\
\sqrt{2} u_{2}(k)
\end{array}\right] .
\end{aligned}
$$




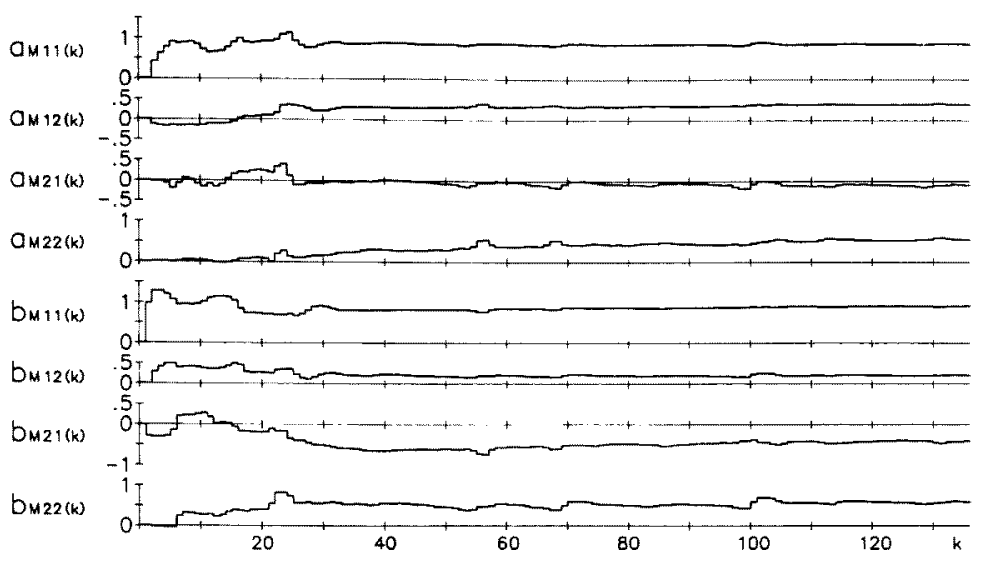

図 5 実数領域での同定特性

Fig. 5. Identification characteristics based on the conventional method.

と記せる。(69)式の同定アルゴリズムと等価になるよ うにダインを $5 \boldsymbol{I}_{2 n}$ とした積分同定アルゴリズムが，

$$
\begin{aligned}
& {\left[\begin{array}{llll}
a_{M 11}(k) & a_{M 12}(k) & b_{M 11}(k) & b_{M 12}(k) \\
a_{M 21}(k) & a_{M 22}(k) & b_{M 21}(k) & b_{M 22}(k)
\end{array}\right]} \\
& =\left[\begin{array}{lll}
a_{M 11}(k-1) & a_{M 12}(k-1) & b_{M 11}(k-1) \\
a_{M 21}(k-1) & a_{M 22}(k-1) & b_{M 21}(k-1)
\end{array} *\right. \\
& \left.* \begin{array}{l}
b_{M 12}(k-1) \\
b_{M 22}(k-1)
\end{array}\right]+\frac{10\left[\begin{array}{l}
\varepsilon_{01}(k) \\
\varepsilon_{02}(k)
\end{array}\right]\left[y_{M 1}(k-1)\right.}{1+10\left\{y_{M 1}^{2}(k-1)\right.} * \\
& * \frac{\left.y_{M 2}(k-1) u_{1}(k-1) u_{2}(k-1)\right]}{\left.+y_{M 2}^{2}(k-1)+u_{1}^{2}(k-1)+u_{2}^{2}(k-1)\right\}}
\end{aligned}
$$

と得られる。ただし，

$$
\left[\begin{array}{l}
\varepsilon_{01}(k) \\
\varepsilon_{02}(k)
\end{array}\right]=\left[\begin{array}{l}
y_{1}(k) \\
y_{2}(k)
\end{array}\right]-\left[\begin{array}{l}
y_{M 01}(k) \\
y_{M 02}(k)
\end{array}\right] \ldots .
$$

でる。図 5 は上記のアルゴリズムに従ってパラメー 夕同定を行ったときの特性である。同定を開始して 135 サンプル経過した時点の推定パラメータの值は それぞれ, $a_{M 11}(135)=0.867, a_{M 12}(135)=0.381$, $a_{M 21}(135)=-0.986, a_{M 22}(135)=0.553, b_{M 11}(135)=$ $0.920, b_{M 12}(135)=0.211, b_{M 21}(135)=-0.403$, $b_{M 22}(135)=0.589$ であり, 推定パラメータとプロセス のパラメータの值の差は大きく，十分な同定がなされ ていない。このシミュレーションで図 4 の 25 サンプ ル目と同程度まで推定パラメータを収束させるには約 850 サンプル必要となる。これらの特性から明らかな ように，提案する複素数領域のパラメー夕同定法を用 いることで高速なパラメー夕同定が実現できる。な
お，実際のシステムへの適用例として，三相 PWM コンバータのパラメータを同定した実験特性について も別に報告している ${ }^{(6)}$ 。

\section{7.むすび}

本論文は，従来実数領域に対して理論展開されてき たMRASのパラメータ同定理論を離散時間系の複素 数領域へ拡張したもので, その成果は以下の上うに要 約できる。

（1）複素数領域に抬張したMRASの比例十積分 同定アルゴリズムを提案した。

（2）複素数領域に拡張した超安定論を導出し，提 案したバラメータ同定アルゴリズムが漱近超安定であ ることを証明した。

（3）複素方程式を実方程式八変換する一方法を示 した。この場合，実数領域で表現すると物理的に等し いパラメータが二つずつ存在するので, 複䒺方程式を 用いることにより同定するパラメータの要素数が $1 / 2$ になる。

（4）複素表現可能なシステムに対して，複素数領 域拉よび実数領域それぞれのMRASに基づいたパラ メータ同定のシミュレーションを行い, その結果, 複 素数領域の MRAS を用いることでパラメータの収束 速度が，実数領域の $1 / 10$ 以下に改善されることを示 した。

なお，本研究について御討論いたたいいた千葉大学工 学部美多 勉教授ならびに㖪業訓練大学校西山清 先生に深く謝意を表す。(平成元年 8 月 23 日受付) 


\section{文献}

（1）宮入：エネルギ一変換工学入門(下) (昭 40) 丸善

(2) I. D.ランダウ・富塚：適応制御システムの理論と実際（昭 56) オーム社

(3) V. M. Popov: Hyperstability of Control Systems (1973) Springer Verlag

(4) B. D. O. Anderson: "A System Theory Criterion for Positive Real Matrices", J. SIAM Control, 5171 (1967)

（う）例えば，児玉・須田：シマテテム制御のためのアトリクス理論 (昭 53) 計測自動制御学会

(6) 竹下・内川・松井：「モデル規範適応同定器を用いた三相 PWM コンパータの電流制御」, 電学論 D, 110, 384 (平 24)

\section{付 録}

\section{1. 定理の証明}

(53)式より次式の関係が得られる。

$$
\begin{aligned}
& \boldsymbol{x}^{*}(k) \boldsymbol{Q}(k) \boldsymbol{x}(k) \\
&=-\{\boldsymbol{A}(k) \boldsymbol{x}(k)\}^{*} \boldsymbol{P}(k+1) \\
& \times\{\boldsymbol{A}(k) \boldsymbol{x}(k)\}+\boldsymbol{x}^{*}(k) \boldsymbol{P}(k) \boldsymbol{x}(k)
\end{aligned}
$$

上式の $\boldsymbol{A}(k) \boldsymbol{x}(k)$ に (51) 式の関係を代入して,

$$
\begin{aligned}
& \boldsymbol{x}^{*}(k) \boldsymbol{Q}(k) \boldsymbol{x}(k) \\
&=-\boldsymbol{x}^{*}(k+1) \boldsymbol{P}(k+1) \boldsymbol{x}(k+1)+\boldsymbol{x}^{*}(k) \boldsymbol{P}(k) \\
& \times \boldsymbol{x}(k)+\boldsymbol{u}^{*}(k+1) \boldsymbol{B}^{*}(k) \boldsymbol{P}(k+1) \boldsymbol{x}(k+1) \\
&+\boldsymbol{x}^{*}(k+1) \boldsymbol{P}(k+1) \boldsymbol{B}(k) \boldsymbol{u}(k+1) \\
&-\boldsymbol{u}^{*}(k+1) \boldsymbol{B}^{*}(k) \boldsymbol{P}(k+1) \boldsymbol{B}(k) \boldsymbol{u}^{*}(k+1)
\end{aligned}
$$

が得られる。更に上式の右辺第 3,4 項の $x(k+1)$

へ(51) 式を代入し, 両辺に $\boldsymbol{u}^{*}(k+1) \boldsymbol{S}^{*}(k) \boldsymbol{x}(k)$ $+x^{*}(k) \boldsymbol{S}(k) \boldsymbol{u}(k+1)+\boldsymbol{u}^{*}(k+1) \boldsymbol{R}(k) \boldsymbol{u}(k+1)$ を加元 て整理すると次式となる。

$$
\begin{aligned}
x^{*}( & (k) \boldsymbol{Q}(k) \boldsymbol{x}(k)+\boldsymbol{u}^{*}(k+1) \boldsymbol{S}^{*}(k) \boldsymbol{x}(k)+\boldsymbol{x}^{*}(k) \\
& \times \boldsymbol{S}(k) \boldsymbol{u}(k+1)+\boldsymbol{u}^{*}(k+1) \boldsymbol{R}(k) \boldsymbol{u}(k+1) \\
= & -\boldsymbol{x}^{*}(k+1) \boldsymbol{P}(k+1) \boldsymbol{x}(k+1) \\
& +\boldsymbol{x}^{*}(k) \boldsymbol{P}(k) \boldsymbol{x}(k)+\boldsymbol{u}^{*}(k+1) \\
& \times\left\{\boldsymbol{B}^{*}(k) \boldsymbol{P}(k+1) \boldsymbol{A}(k)+\boldsymbol{S}^{*}(k)\right\} \boldsymbol{x}(k) \\
& +\left[\boldsymbol { u } ^ { * } ( k + 1 ) \left\{\boldsymbol{B}^{*}(k) \boldsymbol{P}(k+1) \boldsymbol{A}(k)\right.\right. \\
& \left.\left.+\boldsymbol{S}^{*}(k)\right\} \boldsymbol{x}(k)\right]^{*}+\boldsymbol{u}^{*}(k+1) \\
& \times\left\{\boldsymbol{B}^{*}(k) \boldsymbol{P}(k+1) \boldsymbol{B}(k)+\boldsymbol{R}(k)\right\} \boldsymbol{u}(k+1)
\end{aligned}
$$

(52)，(54)，(55)式を用いて変形すると，

$$
\begin{aligned}
& \boldsymbol{v}^{*}(k+1) \boldsymbol{u}(k+1)+\boldsymbol{u}^{*}(k+1) \boldsymbol{v}(k+1) \\
&= \boldsymbol{x}^{*}(k+1) \boldsymbol{P}(k+1) \boldsymbol{x}(k+1) \\
&-\boldsymbol{x}^{*}(k) \boldsymbol{P}(k) \boldsymbol{x}(k)+\left[\boldsymbol{x}^{*}(k) \boldsymbol{u}^{*}(k+1)\right] \\
& \times\left[\begin{array}{ll}
\boldsymbol{Q}(k) & \boldsymbol{S}(k) \\
\boldsymbol{S}^{*}(k) & \boldsymbol{R}(k)
\end{array}\right]\left[\begin{array}{l}
\boldsymbol{x}(k) \\
\boldsymbol{u}(k+1)
\end{array}\right]
\end{aligned}
$$

が得られる。上式を $k$ について 0 $k_{1}$ まで辺々加元， 両辺のトレースをとり，(56)式の関係を考慮すると，

$$
\begin{gathered}
\sum_{k=0}^{k_{1}} \operatorname{tr}\left\{\boldsymbol{v}^{*}(k+1) \boldsymbol{u}(k+1)+\boldsymbol{u}^{*}(k+1) \boldsymbol{v}(k+1)\right\} \\
\geqq-\operatorname{tr}\left\{\boldsymbol{x}^{*}(0) \boldsymbol{P}(0) \boldsymbol{x}(0)\right\} \quad \cdots \cdots \cdots \cdots \cdots(57)
\end{gathered}
$$

(57)式が得られ，定理が成り立つ。

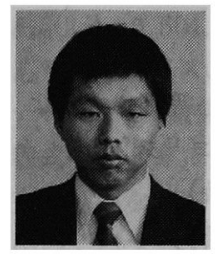

\section{竹 下隆 晴 (正員)}

昭和 34 年 8 月 23 日生。 59 年 3 月名古屋工業大学大学院修士課程修 了。同年 4 月岐阜工業高等専門学校 電気工学科助手, 平成 2 年 4 月同講 師, 現在に至る。工学博士。主として, 交流電動機お よび整流回路のプロセッサ制御の研究に従事。計測自 動制御学会会員。

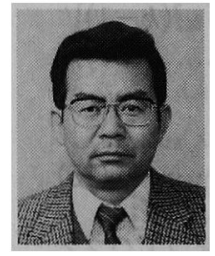

松井信 行 (正員)

昭和 18 年 5 月 7 日生。 43 年 3 月 名古屋工業大学大学院修士課程修 了。同年 4 月同大学助手, 同講師, 同助教授を経て, 60 年 4 月同電気 情報工学科教授, 現在に至る。工学博士。制御理論に よる電動機制御系の解析と設計, 電動機の計算機制御 の研究に従事。計測自動制御学会, IEEE 会員。 